\title{
REPRESENTATIONS OF FUNDAMENTAL GROUPS OF ALGEBRAIC MANIFOLDS AND THEIR RESTRICTIONS TO FIBERS OF A FIBRATION
}

\author{
JÜRGEN Jost AND KANG ZuO
}

\section{Introduction}

We consider a surjective morphism $f: X \rightarrow Y$ from a smooth projective variety $X$ onto a smooth projective variety $Y$ with connected fibers, henceforth called a fibration for short. Typically, if a certain geometric object on $X$ like a cohomology class has a certain property, then its restriction to a smooth fiber of $f$ trivially satisfies the same property. The converse question is of more interest: if the restriction of such a geometric object to a smooth fiber enjoys a certain property, is this property also valid for the object on $X$ itself? A prototype is the geometric version of the $(p, q)$ component theorem of Griffiths saying that if a class $H^{k}(X, \mathbb{C})$ is of pure Hodge type $(p, q)$ at some smooth fiber $f^{-1}(y)$, then it has Hodge type $(p, q)$ at any smooth fiber.

In the so-called nonabelian cohomology, instead of classes in $H^{1}(X, \mathbb{C})$, one considers representations $\rho: \pi_{1}(x) \rightarrow G$ into some linear algebraic group $G$. In the same way as one associates a harmonic form to a cohomology class, one finds a $\rho$-equivariant harmonic map $h: X \rightarrow G / K$ into the symmetric space of noncompact type obtained as a homogeneous space for $G$. This harmonic map turns out to be pluriharmonic, meaning that its restriction to any subvariety is harmonic itself. We may thus reformulate the question indicated in the title of our paper, namely what one can infer about a representation of $\pi_{1}(X)$ if one knows a relevant property of the induced representation on $\pi_{1}\left(f^{-1}(y)\right)$, or, more generally, of the one on $\pi_{1}(Z), Z$ a generic subvariety of $X$, as the question of what we can deduce about a pluriharmonic map from its restriction to $f^{-1}(y)$ or $Z$. Let us start with some easy observations in this direction before formulating our actual results. A harmonic map into $G / K$ is constant on any rational variety. On one hand this implies that a pluriharmonic map $h$ into $G / K$ is an invariant of the birational class of $X$, as has been observed by many people, but on the other hand this tells us that we cannot deduce information about $h$ from its restriction to rational subvarieties. In a more positive direction, if we have a smooth map $g: X \rightarrow G / K$ whose restriction to any fiber $f^{-1}(y)$

Received October 5, 1999.

Revised version received August 25, 2000.

The second author was supported by a Heisenberg fellowship of the DFG. 
is harmonic, and if we are in a situation where the image of the fibers is sufficiently large so that harmonic map uniqueness holds (this is the case if there are no parallel vector fields along the image), then $g$ itself must be harmonic. In particular, in that situation if we have homomorphisms $\rho: \pi_{1}\left(f^{-1}(y)\right) \rightarrow G$ on the fundamental groups of the fibers, then these homomorphisms induce a unique homomorphisms $\rho: \pi_{1}(X) \rightarrow G$. An essential point of the present note consists in results that refine the preceding simple observation. We shall only need the weaker assumption that the harmonic maps on the fibers are nonconstant, or equivalently that the representations on $\pi_{1}\left(f^{-1}(y)\right)$ are nontrivial, plus the assumption that the image of $\pi_{1}(X)$ is Zariski dense. Then the representation of $\pi_{1}(X)$ cannot be deformed with the representation on $\pi_{1}\left(f^{-1}(y)\right)$ kept fixed (see Thm. 2b below). We also study a similar situation where instead of the fibers of a holomorphic map we consider a subvariety $Z$ whose fundamental group surjects onto the one of $X$. Again, in that case, the representation on $Z$ locally determines the one on $X$ (see Thm. 2a). Finally, we study how other properties like coming from a variation of Hodge structures or arithmeticity on the fibers of a holomorphic map induce the corresponding ones on $X$ (Thm. 1 and $2 \mathrm{c}$, resp.).

Remark. We wrote up and circulated the first version of the present paper in December 1998. After we had circulated our result, also a preprint by Katzarkov and Pantev appeared where they proved a somewhat weaker result by a different method. This weaker result had already been announced by them earlier.

\section{Results and proofs}

Theorem 1. Let $f: X \rightarrow Y$ be a fibration and $Z=\cup_{i=1}^{m} Z_{i}$ be a reduced fibre of $f$ with smooth irreducible components. Suppose $\rho: \pi_{1}(X) \rightarrow G$ is a Zariski dense representation into an almost simple algebraic group $G$ and does not factor through $f$. If the restriction $\left.\rho\right|_{Z_{i}}, 1 \leq i \leq m$ comes from $\mathbb{Z}$-variations of Hodge structure, then $\rho$ itself comes from $\mathbb{Z}$-variations of Hodge structure.

We say a representation $\rho: \pi_{1}(X) \rightarrow G$ factors through a fibration $f: X \rightarrow$ $Y$, if there exists a finite etale covering with a blowing up $e: X^{\prime} \rightarrow X$ and a representation $\tau: \pi_{1}\left(Y^{\prime}\right) \rightarrow G$, where $Y^{\prime}$ is the base of the Stein-factorisation $f^{\prime}: X^{\prime} \rightarrow Y^{\prime}$ of $f e: X^{\prime} \rightarrow X \rightarrow Y$, such that $e^{*}(\rho)=f^{\prime *}(\tau)$.

Remark 1. The following consideration was pointed out to the authors by $\mathrm{C}$. Simpson. We can replace $\rho$ by a section of the relative Betti space $M_{B}(X / Y, G)$ which is flat with respect to the nonabelian Gauss-Manin connection introduced by Simpson [S2]. W A flat section glues to a global representation $\tilde{\rho}^{\prime}$ on the covering $\tilde{X}^{\prime} \rightarrow X$ corresponding to $\pi_{1}(X) \rightarrow \pi_{1}(Y) \rightarrow 1$. The decktransformation group $\pi_{1}(Y)$ acts on $M_{B}\left(\tilde{X}^{\prime}, G\right)$. If $G$ is almost simple and the image of $\rho$ is Zariski dense, then we can show that $\rho^{\prime}$ can be descent to a representation $\rho: \pi_{1}\left(X^{\prime}\right) \rightarrow G$, where $X^{\prime} \rightarrow X$ is a finite etale cover. In a forthcoming 
paper [JLZ] we shall prove a general version of Theorem 1 for the case, where $f: X \rightarrow Y$ is a morphism between two quasi-projective varieties.

Theorem 1 is an easy consequence of Simpson's theorem about when a representation will come from variations of Hodge structure and Theorem 2 below, which can be considered as a type of Lefschetz hyperplane theorem for possibly singular subvarieties, whose fundamental groups surjects onto $\pi_{1}(X)$, or for subvarieties arising as fibres of fibrations.

Theorem. (Simpson, Corollary 4.2, [S1]) The representations of $\pi_{1}(X)$ which come from complex variations of Hodge structure are exactly the semisimple ones which are fixed by the action of $\mathbb{C}^{*}$.

Hitchin originally defined this action in the form of an action of $U(1) \subset \mathbb{C}^{*}$ $[\mathrm{H}]$.

Let $Z=\cup_{i=1}^{m} Z_{i} \subset X$ be a subvariety and suppose that all irreducible components are smooth. Given a representation $\rho \in M_{B}(X, G)$, the pull back via $Z_{i} \hookrightarrow X$ defines a point $\left.\rho\right|_{Z_{i}} \in M_{B}\left(Z_{i}, G\right)$. Hence, that defines a morphism

$$
r: M_{B}(X, G) \rightarrow \prod_{i=1}^{m} M_{B}\left(Z_{i}, G\right) .
$$

We may consider $G \subset S L_{n}$ as a group scheme defined over some number field and with a fixed integral structure (for instance, induced by $S L_{n}$ ). So, the morphism $r$ is clearly also defined over some number field.

Theorem 2.

a) Suppose that the homomorphism $\pi_{1}(Z) \rightarrow \pi_{1}(X)$ is surjective. Then the preimage of $r$ over any point consists of finitely many points only.

b) Suppose that $Z=\cup Z_{i}$ is a reduced fibre of a surjective morphism $f$ : $X \rightarrow Y$ with connected fibres, and that $\rho: \pi_{1}(X) \rightarrow G$ is Zariski dense and does not factor through $f$. Then $\rho \in r^{-1}(r(\rho))$ is an isolated point.

c) Let $Z=\cup_{i=1}^{m} Z_{i}$ and $\rho$ be the same as in b). If the restriction $\left.\rho\right|_{Z_{i}}, 1 \leq$ $i \leq m$ is valued in the ring of the algebraic integers of a number field, then this also holds for $\rho$ itself.

Proof of Theorem 1. Applying Simpson's theorem, the action of $\mathbb{C}^{*}$ fixes $r(\rho)$. Since the action of $\mathbb{C}^{*}$ fixing $\left.r(\rho)\right)$ commutes with $r$, the fibre $r^{-1}(r(\rho)$ is fixed by the $\mathbb{C}^{*}$-action. Since $\rho \in r^{-1}(r(\rho))$ is an isolated point by b) in Theorem 2 , $\rho$ is a fix point of the $\mathbb{C}^{*}$-action. Applying Simpson's theorem again, $\rho$ comes from complex variations of Hodge structure. The integral property of $\rho$ follows from c) in Theorem 2.

Proof of Theorem 2 a). Since the morphism

$$
r: M_{B}(X, G) \rightarrow \prod_{i} M_{B}\left(Z_{i}, G\right)
$$

is defined over some number field, we only need to check the property a) for any point $\tau=\left(\tau_{1}, \ldots, \tau_{m}\right) \in \prod_{i=1}^{m} M_{B}\left(Z_{i}, G\right)$, valued in some number field $K$. 
Since $\pi_{1}\left(Z_{i}\right), 1 \leq i \leq m$ is a finitely presented group, we may find a prime ideal $p$ of $\mathcal{O}_{K}$ such that $\tau_{i}\left(\pi_{1}\left(Z_{i}\right)\right) \subset G\left(\mathcal{O}_{K_{p}}\right), 1 \leq i \leq m$, where $K_{p}$ is the local field at the place $p$.

Consider now the morphism $r$ defined over $K_{p}$. If the statement a) were not true, then $r^{-1}(\tau)$ would contain a positive dimensional component $C$. Hence, we may find some representation $\rho \in C$ which is valued in some finite extension of $K_{p}$ and is $p$-unbounded. Notice that $r(\rho)$ is $p$-bounded.

Now let

$$
u_{\rho}: \tilde{X} \rightarrow \triangle\left(G\left(L_{p}\right)\right)
$$

denote the $\rho$-equivariant pluriharmonic map into the corresponding BruhatTits building. The existence of such harmonic maps is shown in [GS]. Since $\rho$ is $p$-unbounded, $u_{\rho}$ is not constant.

On the other hand, the surjectivity of $\pi_{1}(Z) \rightarrow \pi_{1}(X)$ and the $p$-boundedness of $r(\rho)$ imply that $u_{\rho}$ is constant. The following argument can be found in [LR].

Since $\pi_{1}(X) \rightarrow \pi_{1}(Z)$ is surjective, the preimage $\tilde{Z}=\cup \tilde{Z}_{i}$ is connected. The restriction $\left.u_{\rho}\right|_{\tilde{Z}_{i}}$ is just the corresponding equivariant harmonic map of $\tau_{i}$ and is constant, since $\tau_{i}$ is $p$-bounded. Hence, $u_{\rho}(\tilde{Z})$ is a point $q$. Therefore, the action of $\rho\left(\pi_{1}(X)\right)$ also fixes $q$. This implies that $u_{\rho}(\tilde{X})=q$. A contradiction.

Proof of b). We may find a stratification on

$$
r: M_{B}(X, G) \rightarrow \prod_{i=1}^{m} M_{B}\left(F_{i}, G\right)
$$

which is defined over some number field and such that:

i) $r\left(M_{B}(X, G)_{j}\right)=\left(\prod_{i=1}^{m} M_{B}\left(F_{i}, G\right)\right)_{j}$ and

ii) $r: M_{B}(X, G)_{j} \rightarrow\left(\prod_{i=1}^{m} M_{B}\left(F_{i}, G\right)\right)_{j}$ is flat.

We want to show that if $\rho \in r^{-1}(r(\rho))$ is not an isolated point, then $\rho$ factors through $f$ after passing to a finite etale covering and a blowing up $X^{\prime} \rightarrow X$.

Using the above stratification we may first show that property for those $\rho^{\prime}$, who are in the same strata as $\rho$ and valued in some number field. If all such $\rho^{\prime}$ factor through $f$, then $\rho$ also factors through $f$.

Suppose $\rho$ is valued in some number field $K$. By the same reason as explained in the proof of a), we may find a local field $K_{p}$, such that $r(\rho)$ is valued in $\mathcal{O}_{K_{p}}$.

If $\rho \in r^{-1}(r(\rho))$ were not an isolated point, then we would find an irreducible curve $\rho \in C \subset r^{-1}(r(\rho))$ that contains infinitely many $p$-unbounded and Zariski dense representations $\rho_{i}: \pi_{1}(X) \rightarrow G\left(L_{p, i}\right)$, where $L_{p, i}$ is a finite extension of $K_{p}$.

Let

$$
u_{\rho_{i}}: \tilde{X} \rightarrow \triangle\left(G\left(L_{p, i}\right)\right)
$$


denote the $\rho_{i}$-equivariant pluriharmonic map into the Bruhat-Tits building. $u_{\rho_{i}}$ is not constant, since $\rho_{i}$ is not $p$-bounded.

Consider the pulled back fibration

$$
\tilde{f}: \tilde{X} \rightarrow \tilde{Y}
$$

We have

Claim $1 u_{\rho_{i}}$ factors through $\tilde{f}$.

Proof of Claim 1. The differential $d^{\prime} u_{\rho_{i}}$ is a collection of holomorphic 1-forms $\theta_{i}$ on a finite ramified covering $X^{s} \rightarrow X$. The pull back of $\theta_{i}$ to the corresponding fibre $F^{s}=\cup_{i=1}^{m} F_{i}^{s}$ is zero, since $r(\rho)$ is $p$-bounded and $\left.u_{\rho_{i}}\right|_{F_{i}}, 1 \leq i \leq m$ is constant.

The pull back of $\theta_{i}$ to any fibre of $f^{s}$ is also zero, since any closed 1-cycle on a fibre of $f^{s}$ is homotopic to some 1-cycle in $F^{s}$ and the integration of $\theta_{i}$ on any closed 1-cycle on a fibre of $f^{s}$ is zero. Therefore, we see that $u_{\rho_{i}}$ factors through $\tilde{f}$.

Claim 2 Let $F_{0}:=f^{-1}\left(y_{0}\right)$ be a smooth fibre of $f$. Then $\rho_{i}\left(\pi_{1}\left(F_{0}\right)\right)$ is a finite subgroup of $G\left(K_{p}\right)$.

Proof of Claim 2. Pulling back $f$ to the universal coverings, by Claim 1 the harmonic map $u_{\rho_{i}}$ factors through $\tilde{f}$,

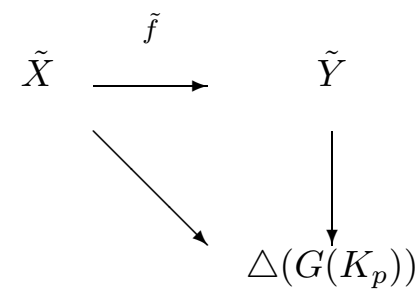

Fixing a base point $x_{0} \in F_{0}$, let $\Gamma=: \operatorname{im}\left(\pi_{1}\left(F_{0}, x_{0}\right) \rightarrow \pi_{1}\left(X, x_{0}\right)\right)$. We first want to show that $\Gamma$ fixes a unbounded subset in $\triangle\left(G\left(L_{p, i}\right)\right)$.

Let $X_{0} \subset X$ be the Zariski open set, such that the map $f: X_{0} \rightarrow Y_{0}$ is regular. We denote by $\tilde{X}_{0} \rightarrow X_{0}$ the universal covering of $\tilde{X}_{0}$.

The subgroup $\Gamma \subset \pi_{1}\left(X_{0}, x_{0}\right)$ operates on a connected component $\tilde{F}_{0,0}$ of the preimage $\tilde{F}_{0} \subset \tilde{X}_{0}$. Since the harmonic map $u_{i}$ is $\rho$-equivariant and factors through the fibration $\tilde{f}: \tilde{X}_{0} \rightarrow \tilde{Y}_{0}$ to an equivariant harmonic map

$$
v_{i}: \tilde{Y}_{0} \rightarrow \triangle\left(G\left(L_{p, i}\right)\right),
$$

$\rho_{i}(\Gamma)$ fixes the image $u_{i}\left(\tilde{F}_{0,0}\right)=v_{i}\left(\tilde{y}_{0,0}\right)=: z_{0,0}$.

If $\tilde{F}_{0, j}$ is another connected component of $\tilde{F}_{0}$, then there exists an element $g_{j} \in \pi_{1}\left(X_{0}, x_{0}\right)$, such that $g_{i}\left(\tilde{F}_{0,0}\right)=\tilde{F}_{0, j}$. So, the conjugation $g_{j} \Gamma g_{j}^{-1}$ operates on $\tilde{F}_{0, j}$, and by the same reason as above $\rho_{i}\left(g \Gamma g^{-1}\right)$ fixes $u_{i}\left(\tilde{F}_{0, j}\right)=$ $v_{i}\left(\tilde{y}_{0, j}\right):=z_{0, j}$. 
Considering the exact sequence of the homotopy groups (coming from the definition of $\Gamma$ )

$$
1 \rightarrow \Gamma \rightarrow \pi_{1}\left(X_{0}, x_{0}\right) \rightarrow \pi_{1}\left(Y_{0}, y_{0}\right) \rightarrow 1,
$$

we see in particular that $\Gamma \subset \pi_{1}\left(X_{0}, x_{0}\right)$ is a normal subgroup. Hence, $\rho_{i}(\Gamma)=\rho_{i}\left(g_{j} \Gamma g_{j}^{-1}\right)$ fixes $z_{0, j}, \forall j \in J$.

Now we show that the subset $Z:=\left\{z_{0, j}\right\}_{j \in J} \subset \triangle\left(G\left(L_{p, i}\right)\right)$ is unbounded. Since each different point in the subset $\left\{y_{0, j}\right\}_{j \in J} \subset \tilde{Y}_{0}$ is contained in a different fundamental domain $D_{j} \subset \tilde{Y}_{0}$ and the images $v_{i}\left(D_{j}\right) \ni z_{0, j}, j \in J$ are uniformly bounded (they are permuted by $\rho_{i}\left(\pi_{1}\left(X_{0}, x_{0}\right)\right)$ as isometry), together with the unboundedness of $v_{i}\left(\tilde{Y}_{0}\right)$ this implies that $Z$ must be an unbounded subset in $\triangle\left(G\left(L_{p, i}\right)\right)$.

We want to show further that $\rho_{i}(\Gamma)$ fixes a point on the boundary of $\triangle\left(G\left(L_{p, i}\right)\right)$. Since $Z \subset \triangle\left(G\left(L_{p, i}\right)\right)$ is an unbounded subset, the convex subcomplex generated by $Z$ contains at least one geodesic line $L$. Since $\rho_{i}(\Gamma)$ fixes $Z$ pointwisely, $\rho_{i}(\Gamma)$ fixes this convex subcomplex pointwisely. Hence, $\rho_{i}(\Gamma)$ fixes $L$ and the point on the boundary of $\triangle\left(G\left(L_{p, i}\right)\right.$ defined by $L$.

That shows that $\rho_{i}(\Gamma)$ is contained in a parabolic subgroup of $G$. In particular, it is not Zariski dense in $G\left(L_{p, i}\right)$. Furthermore, the exact sequence of the homotopy groups above shows that the Zariski closure of $\rho_{i}(\Gamma)$ is a normal algebraic subgroup in $G\left(L_{p, i}\right)$. Since $G\left(L_{p, i}\right)$ is almost simple, $\rho_{i}(\Gamma)$ must be finite. Claim 2 is proved.

By restriction to $F_{0}$ we get a family of representations

$$
\rho_{t}: \pi_{1}\left(F_{0}\right) \rightarrow G\left(K_{p}\right), \quad t \in C .
$$

By Claim $2 \rho_{i}\left(\pi_{1}\left(F_{0}\right)\right) \subset G\left(K_{p}\right), i \in I$ is not Zariski dense. Since $I$ is infinite and $C$ is irreducible, the subset $\left\{\left.\rho_{i}\right|_{F_{0}}\right\}_{i \in I} \subset C$ is Zariski dense. Since $G$ is an almost simple group, the Zariski density of representations is a Zariski open condition in the space of representations. That shows $\rho\left(\pi_{1}\left(F_{0}\right)\right) \subset G\left(K_{p}\right)$ is not Zariski dense, too. By the same reason as in Claim 2, $\rho\left(\pi_{1}\left(F_{0}\right)\right) \subset G\left(K_{p}\right)$ is a finite subgroup.

Since $\rho\left(\pi_{1}(X) \subset G\right.$ is residually finite, one may find a finite etale covering with a blowing up $X^{\prime} \rightarrow X$ such that the pull back of $\rho$ factors through the Stein factorisation of $f: X^{\prime} \rightarrow Y$. So, this leads to a contradiction to our assumption in b).

Proof of c. Since $r(\rho)$ is valued in some number field $K$ and $\rho \in r^{-1}(r(\rho))$ is an isolated point, $\rho$ is valued in some finite extension $L \supset K$. Now as $r(\rho)$ is bounded with respect to any prime ideal of $\mathcal{O}_{K}$, the same argument in b) shows that $\rho$ is also bounded with respect to any prime ideal of $\mathcal{O}_{L}$. 


\section{References}

[G] Griffiths, P. Periods of integrals on algebraic manifolds III, Publ. Math. IHES 38 (1970), 125-180.

[GS] Gromov, M. and Schoen, R. Harmonic maps into singular spaces and p-adic superrigidity for lattices in groups of rank one, Publ. Math. IHES 76 (1992), 165-246.

[H] Hitchin, N.J. The self-duality equations on a Riemann surface, Proc. London Math. Soc. (3), 55 (1987), 59-126.

[H] Jost, J., Li, J-Y. and Zuo, K. Higgs bundles over quasi-compact Kähler manifolds, in preparation.

[LR] Lasell, B. and Ramachandran, M. Observations on harmonic maps and singular varieties, Ann Sci. Ecole Norm. Sup. 29. (1996), 135-148.

[S1] Simpson, C. Higgs bundles and local systems, Publ. Math. IHES 75 (1992), 5-95.

[S2] Simpson, C. The Hodge filtration on nonabelian cohomology, Proc. Symp. Pure Math., Vol 62, Part 2, 217-281.

MaX-Planck-Institut fuer Mathematik in Den Naturwissenschaften Inselstrasse 22 - 26 D-04103 LeIPZIG Germany.

E-mail address: jjost@mis.mpg.de

Department of Mathematics, The Chinese University of Hong Kong, Shatin, N.T. HONG KONG.

E-mail address: kzuo@math.cuhk.edu.hk 